\title{
The influence of quantitative parameters of lubricant oil's on the damaged of car turbochargers - Event Tree Analysis (ETA) method
}

\author{
Maciej Bieliński ${ }^{1}$, Mateusz Bor ${ }^{1}$, Tomasz Borowczyk ${ }^{1}$, Marek Idzior ${ }^{1,}{ }^{*}$, Wojciech Karpiuk ${ }^{1}$, and Rafat Smolec $^{1}$ \\ ${ }^{1}$ Poznan University of Technology, Faculty of Machines and Transport, ul. Piotrowo 3, 60-965 Poznan, Poland
}

\begin{abstract}
The Event Tree Analysis (ETA) is a tool for assessing system and process safety. It facilitates detecting potential risks, as well as relations existing between actions or events. This method explores the path from the initiating event to the outcome (most often representing the immobilization of the tested object), with particular emphasis on intermediate stages affecting the technical condition of the object. Unlike the FTA analyses, which have a deductive nature, the ETA analysis is inductive. The aim of the work was to estimate the probability of occurrence of unwanted events concerning the operation of a car turbocharger. Based on the performed studies, the impact of the standard safety systems on the operation of the component was checked. Afterwards, more effective safety solutions were proposed. Initiating event was distinguished in the system. The occurrence of such an event could result in a complete immobilization of the component. To accomplish the task, it was necessary to use safety barriers aimed at reducing negative effects of initiating events. The calculation of probabilities of occurrences of various effects in each of the cases helped quantify the selected damages to the turbocharger.
\end{abstract}

\section{Introduction and purpose of the study}

To perform the analysis of the system in relation to its damage, it is essential to use unified procedures. The classic approach to determining reliability - as the probability of occurrence of suitability of a system within a given period of time - is replaced by an analysis of possibilities of implementing tasks imposed on the system [1]. Furthermore, in other publications, authors propose to assess the reliability of the process, and not only a single device.

In this paper, to evaluate the possibility of occurrence of the damage, the Event Tree Analysis was used. The damage concerned a component of the engine - a turbocharger, which is a rotary machine and an element of the forced induction of a combustion engine. Due to the operating principle and design features, the device works with many engine systems - intake, exhaust, lubrication systems and also, increasingly more often, with the cooling system.

A multitude of relations, complexity and working conditions are reasons due to which the device is sensitive to disturbances of quality parameters of the working media. Working conditions of turbochargers are highly variable. Such devices, arranged in exhaust systems of combustion engines, are exposed to the following factors (among others):

- high temperatures,

- fluctuations of exhaust gases,
- vibrations caused by the operating engine and the movement of the vehicle point,

- thermal shock caused by fluctuating temperature,

- impact loads.

Continuous improvement of environmental performance of combustion engines is connected with modifications of intake, fuelling, exhaust aftertreatment systems etc. The complexity and the number of moving parts, as well as small fitting tolerances in such systems mean that together with precision devices such as highpressure pumps, injectors or turbochargers, the systems are particularly vulnerable to disturbances of the designed operating parameters of the working media. Devices operate properly as long as the quality and quantity parameters of lubricating oil, combustion air and the cooling liquid are maintained.

Damage to turbochargers in modern combustion engines are a prevalent phenomenon and their sources should be sought in dysfunctions of the following systems: engine lubrication, intake or exhaust systems (exhaust aftertreatment system). The authors' practical expertise proved that, many times, repairs ultimately constist in replacing the turbocharger (without solving the actual problem which initiated the damage). Subsequently, the damage re-occurs, often after a few or a dozen minutes of operation.

Given the large scale of the phenomenon, the aim of the study was specified to determine the probabilities of occurrence of unwanted event, in case of disorders of quality parameters of the working media of the motor

*Corresponding author: marek.idzior@put.poznan.pl 
and turbocharger system. Based on the performed bench tests, the impact of safety systems on the work of the component was studied, and subsequently more effective solutions were proposed. A number of initiating events was distinguished in the system. The occurrence of such events would result in a complete immobilization of the component.

An Event Tree was created for one of such case. To accomplish the task, safety barriers were used. The purpose of the barriers was to reduce negative effects of occurrences of initiating events. The calculation of probability of occurrences of various effects helped quantify the selected damage to the turbocharger.

\section{Research methodology, the applied equipment and the ETA method outlined}

Measurement tests were conducted at a bench designed for testing car turbochargers. The bench includes a combustion engine for generating exhaust gases. Only a stream of hot gases is used to drive the turbocharger. Apart from that the turbocharger operates in an environment isolated from the engine. The bearings are lubricated using an independent lubrication system, including an electric pump, which makes it possible to adjust the whole range of pressure and output. The oil tank ensures that a specific temperature of the medium can be maintained. Compressor air is not used in any way. The system is not connected to the engine intake system. The bench was designed in a way to make it possible to simulate the phenomena affecting the disturbance in quality or quantity parameters of the working media of the engine.

a)

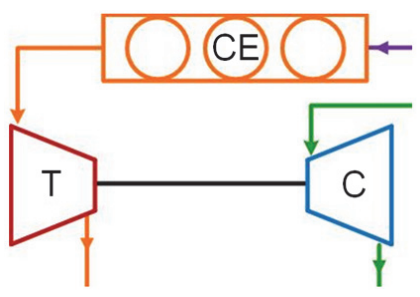

b)

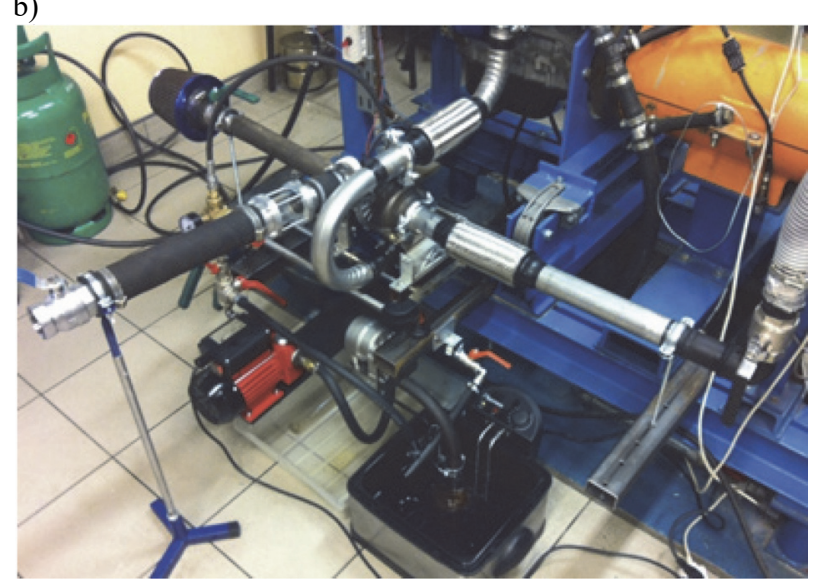

Fig. 1. Test bench with a combustion engine as a gas generator: a) block diagram: $\mathrm{CE}$ - combustion engine, $\mathrm{T}$ - turbine, $\mathrm{C}-$ compressor, b) real view.
The main advantage of the bench is that the test conditions are as close as possible to the real conditions of a turbocharger coupled with an engine. Temperature, pressure and pressure fluctuations are similar to those existing in the exhaust manifold of a supercharged combustion engine. For checking and recording parameters of factors in various branches of the system, temperature sensors and pressure converters were used.

A disadvantage of the system using an engine as the exhaust gases generator is the fact that the energy stream of exhaust gases is strictly connected to the load and the rotational speed, resulting in dissipation of energy in the brake and in overcoming own resistance of the engine.

The bench is equipped with a four-cylinder, naturally aspirated, 16V FIAT SI (Spark Ignition) engine with a displacement of $1.2 \mathrm{dm}^{3}$, with the trademark of MPFI (Fig. 1). The obtainable power is $52 \mathrm{~kW} / 6000 \mathrm{~min}^{-1}$ and the torque is $102 \mathrm{Nm} / 3250 \mathrm{~min}^{-1}$. The used test stand is a multi-directional operation AMX-210/100 with a maximum load power of $100 \mathrm{~kW}$ and a maximum rotational speed of $10,000 \mathrm{~min}^{-1}$. The maximum torque is $240 \mathrm{Nm}$. The engine is equipped with a complete set of instruments for continuous operation, as well as power and torque regulation, making it possible to produce a controlled stream of exhaust gases at given parameters [2].

For the purpose of the study we assumed that the operation of most turbochargers, their operational parameters and their design are not very different between manufacturers or models. The essence of reliability of turbochargers is to take up the problem in a systematic way. It is particularly noteworthy that during the identification and analysis of the damages, these machines should not be considered separately.

Due to the difficulty of modifying connectors for other turbochargers and the need of performing tests on one component, we refrained from using the classical method of assessing reliability and from determining empirical indices of reliability. Instead, we focused on other methods, such as ETA, FTA and FMEA. This article presents an analysis of damage, carried out using the ETA method, which is a part of a doctoral thesis of one of the authors.

The ETA method is a technique for identifying and assessing a sequence of events resulting from an initiating event. The purpose of this analysis is to determine the distribution of potential severity of the risk connected with the initiating event, as well as identifying the impact of the effectiveness of instruments responding to the risk. There are two forms of ETA: pre-occurrence and post-occurrence. The pre-occurrence ETA makes it possible to test the effectiveness of neutralizing the severity of a risk. The post-occurrence ETA is used to analyze the materialized risk and opportunities of managing the level of its severity [3, 4].

According to Oprychał, for the Event Tree method to be applied, it should be assumed that a major malfunction is a result of not just a single occurrence of an initiating event, but the confluence of several events or a series of events. The appearance of a single dysfunction does not generate a chain reaction, nor a serious malfunction. It is not until a sequence of adverse 
conditions appears, causing subsequent damages, that may result in serious malfunctions.

Using the ETA method, one can graphically present a sequence of events and barriers, beginning with initiating a state likely to cause anomalies and resulting in effects arising at each stage of the analysis. Most often, the barriers include safety systems (e.g. an air filter, a $\mathrm{CO}_{2}$ sensor or a fuse), as well as the human factor, which can directly minimize the effects of an occurrence of a malfunction (e.g. a CNC operator or a production line quality inspector). In this method it is assumed that each event in a sequence is a success or a failure (no intermediate states).The probabilities assigned to individual branches in Event Trees are conditional probabilities [5].

When conducting the analysis at each stage, one needs to ask the question "what if?" The operating algorithm is based on analyzing possibilities of evolving of the initiating event.

It is important to accurately determine safety barriers which effectively limit negative effects of the initial event. The ETA method is a logical, divalent tree with the initiating event at the base and a root leading to the event with the most serious effect. Each barrier creates a branch which is also a result of the considered logic state: a success (yes) or a failure (no) depending on whether the given barrier is effective or not. Events arranged in the branches are combinations of sequences of consecutive events.

The result of the tree analysis is the systematization of all possible sequences of events concerning the adopted classification of effects (in case of studying a turbocharger it is the degree of damages, their extent and consequences possibly caused by the faulty component). The ETA technique also makes it possible to calculate the probabilities of occurrence of each group of sequences.

In order to perform the analysis, it is necessary to estimate the probability of occurrence of the initiating events and the probabilities of occurrence of events at specific barriers, obtained from the bench tests. For the case discussed in this article, this step was performed based on one of the most common fault occurring in turbochargers, which was provided to facilities specialized in regenerating components. The additionally applied estimates take into account community interviews with people leading the above facilities. The selected case is presented and discussed in this article.

The ETA analysis was performed with the following steps:

1. Identifying initiating event which could lead to a complete immobilization of the turbocharger - the so-called state of unfitness (in extreme cases of the entire engine),

2. Suggesting alternative solutions for securing the system from critical effects,

3. Creating an Event Tree, Bullets,

4. Estimating the probabilities of occurrence of the initiating event and the occurrence of events at specific barriers,

5. Describing the malfunctions and determining their effects,
6. Calculating the probabilities of occurrence of a given type of effects for the existing and the proposed solutions,

7. Determining existing systems preventing the occurrence of an event or effects leading to the state of unfitness (so called safety barriers).

\section{Results of the study}

Based on the selected cause of damage of turbochargers, we focused on the following initiating event concerning the bearings lubrication system: clogged oil drains out of the center housing.

The selected initiating event is not random. According to information obtained in turbocharger regeneration facilities, it is the most common causes of damage of these types of devices. Therefore, it was decided to analyze the above case with the previously described test bench. Among others, we studied the impact of oil pressure increase in the turbocharger housing on the rotational speed of its shaft and the oil temperature. Tests were performed at the previously described bench. The change of passability (and thus a pressure increase) was simulated with a ball valve located directly behind the oil drain. Using appropriate sensors we recorded the rotational speed of the turbocharger shaft, pressure and oil temperature. We also studied the chemical composition of exhaust gases. Figure 2 presents significant correlations noted during the tests.

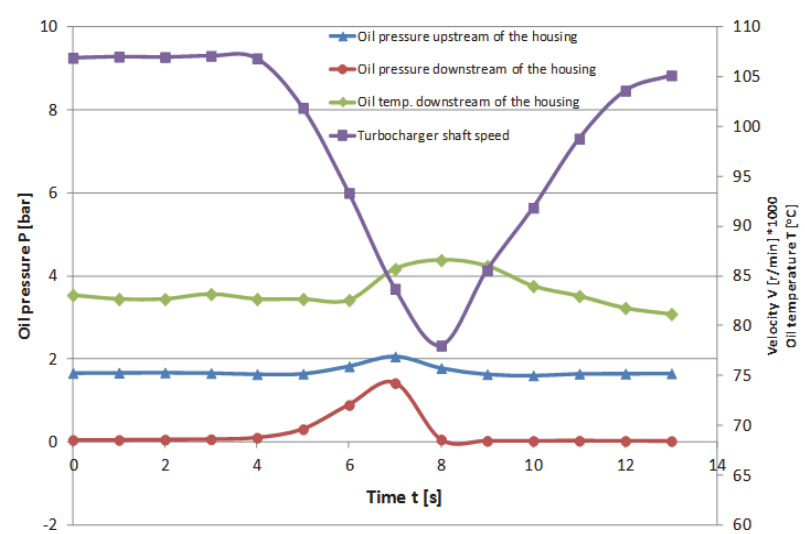

Fig. 2. The impact of oil pressure on the rotational speed of the turbocharger shaft.

The characteristics allows to conclude that oil, after flowing through bearings, is subject to expansion to ambient pressure, and then flows, gravitationally, to the oil sump. Oil flow throttling results in immediate increase in pressure. The pressurized medium disrupts the labyrinth flowing into the turbine chamber, where it is burned, and then flows into the exhaust system. For seal break to happen, it is necessary to produce force which creates resistance also in the system bearings. Moreover, it was noted that oil flow throttling also affects heat dissipation from the bearings in the housing, which certainly has a negative impact on the proper functioning of the device. 
The first analyzed case of a turbocharger damage is a problem of an obstructed oil drain from the housing event A, briefly defined as the "clogged center housing oil drains".

This phenomenon takes place in the oil drain channels, often located at parts of the engine exhaust system which heat up significantly. Some of the most vulnerable elements are large radius bends, narrow passages and mesh screens mounted inside the channels. The Event Tree caused by the reason is shown in Fig. 3.

The engine lubrication system contains the following safety barriers:

$\mathrm{B}$ - oil filter,

$\mathrm{C}-$ actions taken by a service technician.

Effects of different $\mathrm{S}$ events have the following interpretation:

$\mathrm{S}_{1}$ - The oil filter stopped substances hindering the flow in oil channels. The intervention is to replace the filter.

$\mathrm{S}_{2}$ - A serviceman performed oil change within the period recommended by the manufacturer (or before the end of this period). For the barrier to be effective, it must be assumed that the used oil has parameters suitable for the given engine, and the engine operating parameters, as well as the driving conditions of the car, were compliant with the manufacturer's guidelines [6].

$\mathrm{S}_{\mathrm{X}}$ - This is a critical effect which causes the immobilization of the turbocharger and may affect the proper operation of the engine. In case of effect SX repair or replacement of the turbocharger is necessary. Oil drain channels should be unclogged (sometimes aftermarket parts manufacturers make modifications involving the removal of the mesh screen from the tubing, which in extreme cases, in original parts, can cause blocking of the oil drain). In addition, the inside of the engine should be flushed and oil should be replaced (together with the filter).

Quantitative analysis of the success branch (to avoid a critical event) is described as $\mathrm{P}\left(\mathrm{S}_{1,2,3 \text { or } 4}\right)$ and the failure branch $1-\mathrm{P}\left(\mathrm{S}_{1,2,3}\right.$ or 4$)$. Graphical values are shown in Fig. 3.

The probabilities of occurrence of a given type of effects in quantitative terms are as follows:

$$
\begin{gathered}
\mathrm{P}(\mathrm{S} 1)=\mathrm{P}(\mathrm{A}) \bullet \mathrm{P}(\mathrm{B}) \\
\mathrm{P}(\mathrm{S} 2)=\mathrm{P}(\mathrm{A}) \cdot[1-\mathrm{P}(\mathrm{B})] \bullet \mathrm{P}(\mathrm{C}) \\
\mathrm{P}(\mathrm{SX})=\mathrm{P}(\mathrm{A}) \cdot[1-\mathrm{P}(\mathrm{B})] \bullet[1-\mathrm{P}(\mathrm{C})]
\end{gathered}
$$

\begin{tabular}{|c|c|c|c|}
\hline $\begin{array}{c}\text { elogged oil drain } \\
\text { channels }\end{array}$ & oil filter & $\begin{array}{c}\text { driver's/service } \\
\text { technician's } \\
\text { action }\end{array}$ & effect \\
\hline
\end{tabular}

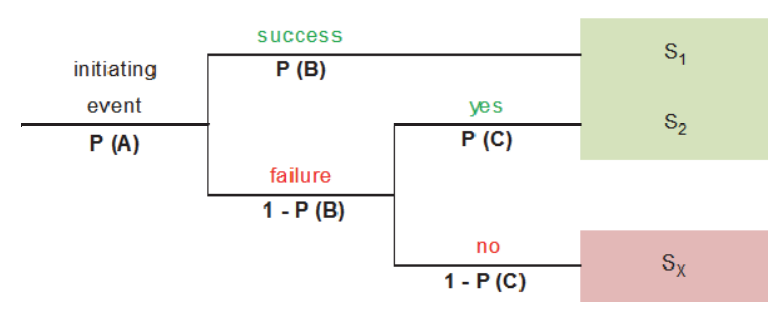

Fig. 3. Event Tree for the initiating event "clogged oil drains out of the center housing“".
The probabilities of occurrence of initiating event $\mathrm{P}(\mathrm{A})$ was determined based on expertise and research. Clogged oil drains from the turbocharger housing are important for proper functioning of the device. As the oil is drained into the oil sump at atmospheric pressure, fluctuation of flow parameters is not uncommon. A well operating crankcase ventilation is particularly important in this case (commonly referred to as "a breather"). Moreover, the practiced solution of placing a mesh screen inside mounting screws (Fig. 4) or oil drain pipes effectively impede the liquid flow.

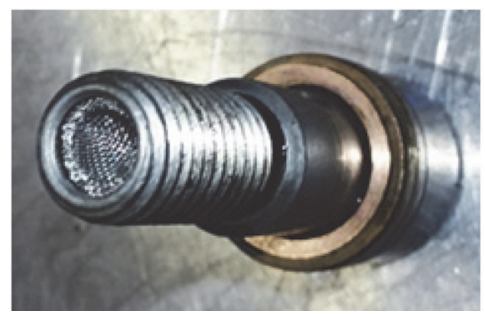

Fig. 4. Screw fixing the drainage hose oil from the turbocharger.

Flow disturbance results in impaired heat transfer and lubrication, and may also cause oil leakage through shaft seals of the turbocharger. During assigning values, taking into account the statistical analysis of the occurrence of the given cause, we assumed the range from " 0 " to " 1 ", wherein " 0 " means no occurrence of the event and "1" a 100\% probability of occurrence. The probability value $\mathrm{P}(\mathrm{A})$ was therefore defined at the level of 0.08 . This means that in $8 \%$ of cases out of the group of all damaged turbocharger, a disrupt in oil flow inside the oil drain channel was noted. This faulty condition, without taking appropriate actions, can lead to the immobilization of the device.

Assigning values to barriers was done similarly to the initiating event. " 0 " means that the barrier does not work, does not affect or does not exist. The value of "1" is equivalent with a $100 \%$ effectiveness of the barrier. The probability values of the event at barrier $\mathrm{P}(\mathrm{B})$ were defined as 0.1 . The oil filter is a barrier as it stops some part of contaminants, yet the phenomenon associated with blocking of flow is also caused by coking of oil (high temperature in the area of oil drain channels) and the aforementioned mesh screens. Oil quality parameters and the percentage of washing additives are also very important. Driver's/service technician's action as barrier $\mathrm{C}$ - relates to the quality and quantity of the lubricating medium in the system. Thus defines checking of the state of oil, caring about intervals between replacing parts and ensuring a proper oil viscosity and lubrication class. Taking into account all functions and capabilities, the parameter was estimated at 0.5 .

Numerical values of probabilities of occurrence of a given type of effects in quantitative terms are as follows: $\mathrm{P}\left(\mathrm{S}_{1}\right)=0.008 ; \mathrm{P}\left(\mathrm{S}_{2}\right)=0.036$. The probability of occurrence of a critical event is equal to: $\mathrm{P}\left(\mathrm{S}_{\mathrm{X}}\right)=$ $=0.036$. When conducting the analysis at each stage, one needs to ask the question "what if?" The operating algorithm is based on analyzing possibilities of evolving of the initiating event. 


\section{Proposed solutions}

In order to reduce the probability of occurrence of a critical event $\mathrm{P}\left(\mathrm{S}_{\mathrm{X}}\right)$, we introduced barriers, which, in the research model, are specific technical solutions applied at the test bench. These were: an oil temperature sensor (indicated as barrier D) located in the turbocharger housing and an oil flow meter (indicated as barrier E) located in the oil drain channel evacuating the oil from the turbocharger housing to the engine oil sump.

The modified Event Trees are marked with the "prim" symbol. For the first described case, concerning the obstructed oil drain from the housing, an analysis shown in Figure 5 was performed.

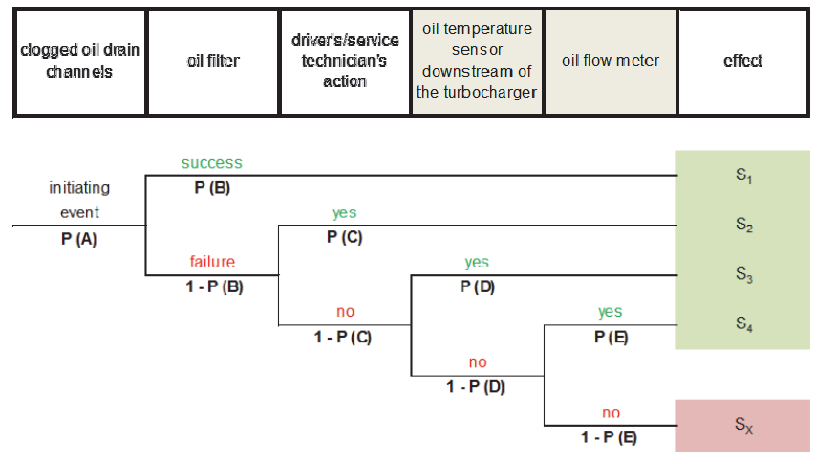

Fig. 5. Event Tree for the initiating event "clogged oil drains out of the center housing".

Probabilities in qualitative terms are analogous to the analyses carried out before introducing changes and for the initiating event equal $\mathrm{P}(\mathrm{A})^{\prime}=0.08$; and for events at specific barriers $\mathrm{P}(\mathrm{B})^{\prime}=0.1 ; \mathrm{P}(\mathrm{C})^{\prime}=0.5$. For the proposed, new barriers, they equal $\mathrm{P}(\mathrm{D})^{\prime}=0.9 ; \mathrm{P}(\mathrm{E})^{\prime}=$ $=0.9$. The values were taken at a $90 \%$ efficiency level, assuming that no solution gives a $100 \%$ certainty. Numerical values of probabilities of occurrence of a given type of effects in quantitative terms are as follows: $\mathrm{P}\left(\mathrm{S}_{1}\right)^{\prime}=0.008 ; \mathrm{P}\left(\mathrm{S}_{2}\right)^{\prime}=0.036 ; \mathrm{P}\left(\mathrm{S}_{3}\right)^{\prime}=0.0324 \mathrm{i} \mathrm{P}\left(\mathrm{S}_{4}\right)^{\prime}=$ $=0.00324$. The probability of occurrence of a critical event is equal to: $\mathrm{P}(\mathrm{SX})^{\prime}=0.00036$.Typing the paper in this format is for the purpose of facilitating the editing process. The forms with text and figures will be printed as delivered. Only in very few exceptional cases will other forms be accepted. the Bibliography (in alphabetical order), e.g.: [1].

\section{Summary and discussion of the results}

The performed analysis, preceded with bench tests, proved that introducing additional safety barriers in the form of sensors, brings measurable benefits. For economic reasons, in mass production, the cost of applying additional sensors and including them in monitoring systems may adversely affect the price of the turbocharger/engine. However, taking into account the benefits, e.g. ones associated with meeting environmental requirements, the proposal presented in the article seems to be justified. In the total balance it makes it possible to significantly reduce the environmental cost (the impact of emissions of harmful substances from exhaust gases during operation) caused by allowing a critical event to occur, while reducing the cost of replacing devices with new ones, as well as the need to look for the cause $[7,8]$. The model proved that the probability of occurrence of a critical event was significantly reduced.

Although the introduced indicator of improving reliability of the system using the same barriers for each case differs significantly, even a difference 90-times higher significantly affects the life of the component. The authors attempted to analyze damage to turbochargers with certain expert assumptions, which can be freely modified, taking input data from research or service repair statistics. As the estimated values may be subject to discussion, the proposed monitoring solutions show clear relations, having a positive reliability and durability impact [9]. The ETA method makes it possible, in a transparent way, to define interrelations occurring between the operation and unfitness of safety systems. Such systems should be taken into account at the stage of designing devices. The paper should be as informative as possible. The research results have to be described in an explicit way. The applied research apparatus and methodology of full value should be introduced precisely. Very extensive literature surveys are not recommended, provided they are not the main target of the paper.

\section{LIDER}

This article was financially supported within the project "New generation of common rail pumps" - Lider/015/273/L5/13/NCBR/2014, implemented within the LIDER Programme, financed by the National Centre for Research and Development, Poland.

\section{References}

1. D. Bobrowski, Metody sieciowe $w$ inżynierii niezawodności I (1999)

2. M. Idzior, W. Karpiuk, M. Bieliński, T. Borowczyk, P. Daszkiewicz, P. Stobnicki, Technika Transportu Szynowego, 3965-3977 (2012)

3. J. Merkisz, J. Mizera, M. Bajerlein, L. Rymaniak, P. Maj, Experimental and Applied Mechanics 518, 102-107 (2014), DOI: 10.4028/www.scientific.net/AMM.518.102

4. blogoryzyku.blogspot.com (2016)

5. K. Szwedzka, J. Gruszka, P. Szafer, IOP Conference Series-Materials Science and Engineering 145, $062007 \quad$ (2016), DOI: 10.1088/1757899X/145/6/062007

6. M. Idzior, M. Bieliński, T. Borowczyk, W. Karpiuk, Logistyka 1129-1139 (2010)

7. M. Idzior, W. Karpiuk, M. Bieliński, T. Borowczyk, Problemy recyklingu 201-207 (2012) 
8. L. Oprychał, K. Fiedler, W. Jankowski, A. Mazurczyk, Metody Komputerowe w Projektowaniu i Analizie Konstrukcji Hydrotechnicznych (1994)

9. M. Orczyk, F. Tomaszewski, B. Czechyra, G.M. Szymanski, Proceedings of the International Congress on Sound and Vibration (Athens, 2016) 